\title{
Chemometrics Pattern Recognition and Calibration Techniques Applied to Volatile Components and Sensory Quality of Kenyan Black Teas
}

\author{
Tetsuo Aishima, ${ }^{1}$ Natsumi TogARI ${ }^{2}$ and Philip O. OwuOR ${ }^{3}$ \\ ${ }^{1}$ Research \& Development Division of Kikkoman Corporation, 399 Noda, Noda, Chiba 278, Japan \\ ${ }^{2}$ Gifu Women's University, 80 Taromaru, Gifu, Gifu 501-25, Japan \\ ${ }^{3}$ Tea Research Foundation of Kenya, PO Box 820, Kericho, Kenya
}

Received April 21, 1995

\begin{abstract}
Multivariate analysis was applied to 35 volatile components and sensory scores of 16 Kenyan black teas made of tea leaves of two clones cultivated at eight areas. Although profiles of volatile components in black teas belonging to a clone deviated according to their harvesting areas, cluster analysis and factor score plots clearly showed differences in the two clones. Multivariate calibration methods provided equations predicting sensory scores using

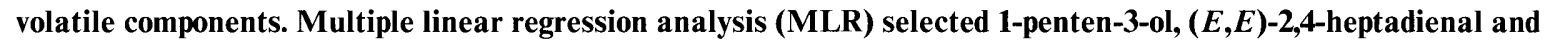
linalool oxide as effective components and the resulting multiple correlation coefficient $(R)$ was 0.914 . The optimum number of principal components indicated by cross-validation was three in the partial least squares (PLS) regression analysis with an $R$ value of 0.946 . A higher correlation of components eluted after linalool, i.e., $\alpha$-cedrene, 3,7-dimethyl-1,5,7-octatrien-3-ol, cedrol and bovolide, to tea quality was suggested by factor loadings of PLS. Principal component regression (PCR) with four principal components showed the lowest $R(0.811)$ among the three calibration methods.
\end{abstract}

Keywords: black tea, aroma, chemometrics, pattern recognition, PLS

Although nearly 500 compounds have been identified in tea volatiles (Yamanishi, 1981; Flament, 1989), research on assessing contributions of individual components to tea aromas was only recently systematically performed (Togari et al., 1995a). Several indices have been proposed to objectively express the aroma quality of black teas based on quantitative gas chromatographic (GC) data of volatile components (Wickremansinghe et al., 1973; Yamanishi et al., 1978; Owuor et al., 1988, Owuor, 1992; Mahanta et al., 1988). Most research has attempted to correlate the ratios of unsaturated aliphatic compounds eluted before linalool to linalool and/ or terpene alcohols eluted after linalool with sensory scores. However, such key volatile components seemed to be intuitively selected or merely based on the experiences of researchers. Of course, all papers have shown the predictability of proposed models for their sample teas but most of them failed to show the fully acceptable reasons for the selection of such key components. The difficulty is understandable when picking a few key components from the large number of volatile components that appeared in capillary GC profiles by simply comparing their peak areas or heights. Recently, Guth and Grosch (1993) found that (Z)-3-hexenal, linalool and $(Z)$-octa-1,5-dien-3-one were highly potent aroma compounds in black tea using the aroma extract dilution methods (AEDA).

Many methodologies in chemometrics have been used to extract useful information on samples or systems from complicated data matrices obtained from instrumental analysis such as capillary GC or various spectrometries (Aishima \& Nakai, 1991). Multiple linear regression analysis (MLR) has been frequently applied to relate instrumental data of food components to sensory evaluation since the late 60's. However, two multivariate calibration techniques such as principal component regression (PCR) analysis and partial least squares (PLS) regression analysis are becoming popular in order to overcome the disadvantages of MLR. Concerning tea research, MLR was used to relate water soluble components to the sensory quality of green tea tastes (Nakagawa et al., 1981). Recently, artificial neural networks (ANN) succeeded in predicting black tea quality based on an HPLC data set (Tomlins \& Gay, 1994). Multivariate statistical analysis was also applied to the near infrared reflectance (NIR) spectra of tea leaves for discriminating black tea qualities (Osborne \& Fearn, 1988) and geographic origins (Grant et al., 1988). Concerning the applications of chemometrics techniques to the volatile components of teas, green tea, Oolong tea and black tea were clearly differentiated based on their volatile components using chemometrics pattern recognition techniques (Togari et al., 1995b). Further, highly predictive equations for their sensory properties were calculated by both MLR and PLS regression but not PCR (Togari et al., 1995a).

In this research, multivariate pattern recognition techniques were applied to the GC data of aroma components in black teas made of tea leaves from two clones cultivated at geographically different areas in Kenya. MLR, PCR and PLS regression analyses were applied to the $\mathrm{GC}$ data in order to establish predictive equations for sensory scores and to determine components contributing to tea quality.

\section{Experimental}

Samples Black teas were made from the leaves of clone 


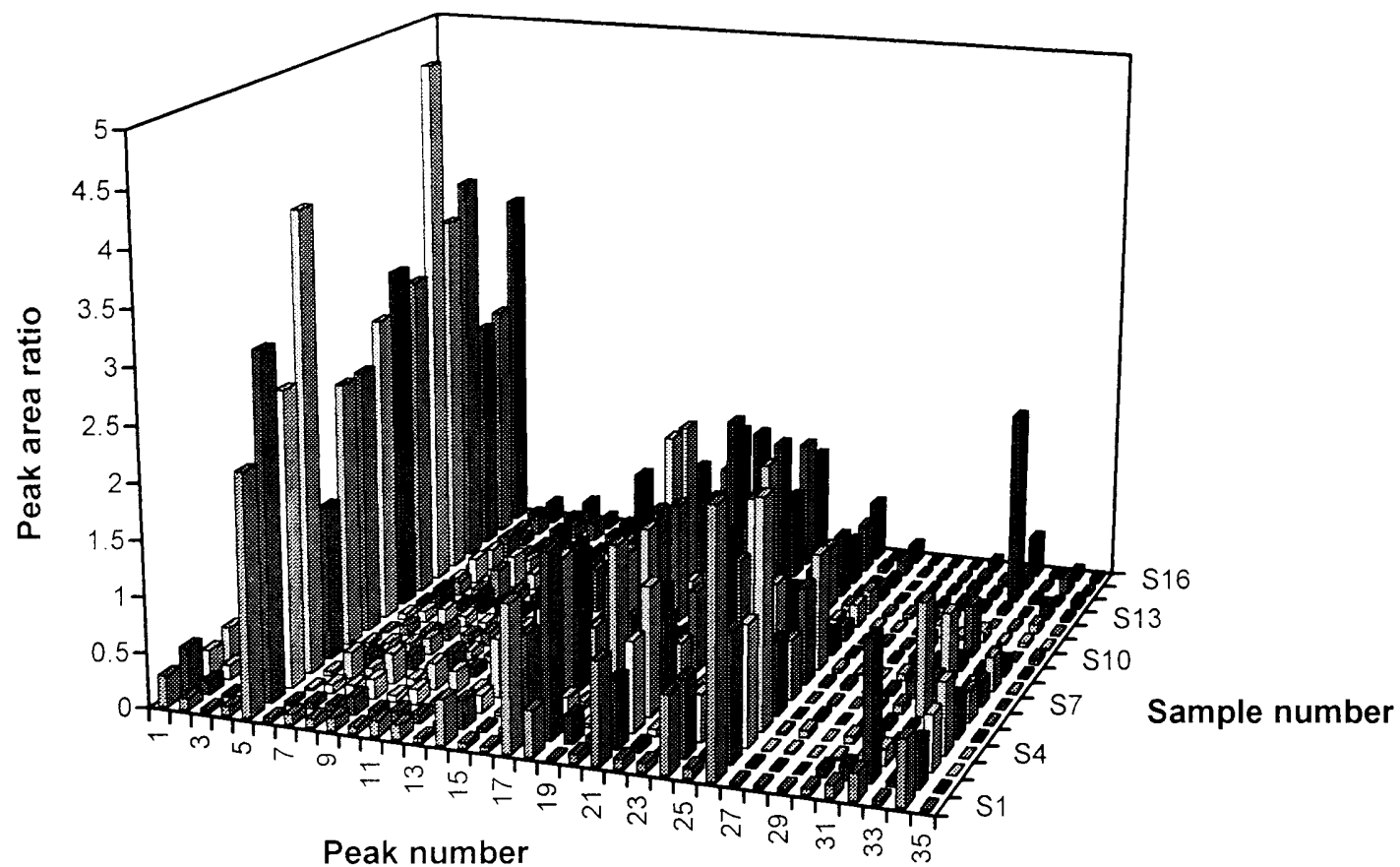

Fig. 1. Gas chromatographic (GC) patterns of aroma components in black teas S1-16 correspond to KR6/8, TI6/8, SI6/8, KN6/8, KG6/8, CH6/8, KI6/8, SO6/ 8, KRTN, TITN, SITN, KNTN, KGTN, CHTN, KITN and SOTN. Peaks 1-35 refer to Table 2.

6/8 and TN 14-3 cultivated at eight different areas in Kenya such as Karirana, Timbell, Sitoi, Kangaita, Kagochi, Changoi, Kaimosi and Sotik (Owuor, 1992). All tea samples were manufactured by a miniature 'crush, tear and curl' (CTC) method (Owuor et al., 1989). The 16 samples thus obtained were abbreviated as follows; Karirana: KR6/8 and KRTN, Timbell: TI6/ 8 and TITN, Sitoi: SI6/ 8 and SITN, Kangaita: KN6/8 and KNTN, Kagochi: KG6/8 and KGTN, Changoi: CH6/8 and CHTN, Kaimosi: KI6/8 and KITN, and Sotik: SO6/8 and SOTN.

$G C$ and sensory data All methodologies regarding sampling, GC analysis and sensory evaluation were described in a previous report (Owuor, 1992). The amounts of volatile components were expressed as the ratios of GC peak areas to those of standard peaks. The quantitative GC data set composed of 35 volatile components in 16 black tea samples (Fig. 1) and their sensory scores were used.

Pattern recognition Factor analysis (FA) and cluster analysis were performed by SPSS for Windows ver. 6.0 (SPSS, Chicago, IL, USA). Cluster analysis was applied to ratios of GC peak areas and their $Z$-scores. The $Z$-transformation standardized means and standard deviations of all variables to 0 and 1.0, respectively, regardless of absolute magnitude of original variables. Euclidian distances and cosines of vectors were used to express the similarities among the $16 \mathrm{GC}$ profiles. A cosine of two vectors corresponded to an angle of the two vectors scattered in the 35 dimensional hyperspace. If two vectors are exactly the same pattern then the angle should be $0^{\circ}\left(\right.$ cosine $\left.0^{\circ}=1.0\right)$. On the contrary, if two vectors do not correlate at all or are orthogonal, then the angle becomes $90^{\circ}$ (cosine $90^{\circ}=0$ ). Centroid and Ward's methods were used to merge the resulting sub-clusters (SPSS; Norusis, 1993). Factors were extracted from the correlation matrix composed of 35 variables using principal component analysis (PCA) and then the factor axes were rotated using the varimax algorithm.

Multivariate calibration Basic models of three multivariate analyses are shown below. When a sensory score and standardized GC peak areas of the $i$ th sample are expressed by $y_{i}$ and $x_{i k}$, the MLR model is expressed by Eq. (1).

$$
y_{i}=b_{0}+b_{1} x_{i 1}+b_{2} x_{i 2}+\ldots+\ldots+b_{m} x_{i m}+e_{i}
$$

In Eq. (1), regression coefficients $\left(b_{i}\right)$, the residual $\left(e_{i}\right)$ and constant $\left(b_{0}\right)$ are calculated using the ordinary least squares method. In this research, stepwise MLR was used by setting $F$ values as 3.84 and 2.9 for selecting and removing the criteria of variables, respectively.

PCR and PLS models are expressed by Eq. 2.

$$
y_{i}=b_{0}+b_{1} t_{i 1}+b_{2} t_{i 2}+\ldots+\ldots+b_{q} t_{i q}+e_{i}
$$

In Eq. (2), $t_{i q}$ is the $q$ th score related to the $i$ th sample. Usually, $q$ is much smaller than $m$. The $p$ th score can be expressed as a linear combination of the original $m$ peaks in the $i$ th black tea sample (Eq. (3)).

$$
t_{i p}=v_{p 1} x_{i 1}+v_{p 2} x_{i 2}+\ldots+\ldots+v_{p m} x_{i m}
$$

In both PCR and PLS, the coefficients of $\left\{v_{p j}\right\}$ are calculated so as to make $\left(v_{p 1}, v_{p 2}, \ldots, v_{p m}\right)$ and $\left(v_{n 1}, v_{n 2}, \ldots\right.$, $v_{n m}$ ) orthogonal. However, there is a significant difference between PLS and PCR. In PCR, $\left\{v_{p j}\right\}$ are calculated only from GC data but both GC and sensory data are used for calculating $\left\{v_{p j}\right\}$ in PLS. Thus, better predictability may be obtained for PLS because PLS can utilize all the information contained in both the GC data and sensory scores.

The most appropriate numbers of the principal components (PC) and PLS components were determined by crossvalidation in PCR and PLS (Sharaf et al., 1986). In crossvalidation, a part of the sample rows was randomly deleted from the data matrix and PCR or PLS was performed for the reduced data matrix. The sensory scores of the deleted samples were predicted using the first PC (PC 1) to calculate the 
Table 1. Combinations of highly correlated peaks.

\begin{tabular}{ccl}
\hline$r$ & Peak number and component & Peak number and component \\
\hline 0.963 & $18: \alpha$-Cedrene & $19: 3$,7-Dimethyl-1,5,7-octatrien-3-ol \\
0.958 & $2: 1$-Penten-3-ol & $7:(Z)$-2-Penten-1-ol \\
0.926 & $26:$ Geraniol & $34:(E)$-Geranic acid \\
0.889 & $2: 1$-Penten-3-ol & $4:(E)$-2-Hexenal \\
0.881 & $18: \alpha$-Cedrene & $32:$ Cedrol \\
0.859 & $12:$ Linalool oxide (cis furanoid) & $14:$ Linalool oxide trans furanoid $)$ \\
0.844 & $18: \alpha$-Cedrene & $33:$ Bovolide \\
0.824 & $7:(Z)$-2-Penten-1-ol & $21:$ Phenylacetaldehyde \\
0.821 & $16:$ Benzaldehyde & $20: \beta$-Cyclocitral \\
0.816 & $3:$ Heptanal & $10:$ Nonanal \\
-0.722 & $1:$ Hexanal & $11:(E)$-2-Hexen-1-ol \\
-0.698 & $30: \beta$-Ionone & $34:$ Bovolide \\
-0.697 & $18: \alpha$-Cedrene & $28:$ Benzyl alcohol \\
-0.649 & $34:(E)$-Geranic acid & $35:$ Indole \\
-0.623 & $10:$ Nonanal & $34:$ Bovolide \\
-0.614 & $1:$ Hexanal & $34:$ Bovolide \\
-0.599 & $21:$ Phenylacetaldehyde & $32:$ Cedrol \\
-0.598 & $25:$ Nerol & $35:$ Indole \\
-0.596 & $8:$ Hexanol & $29: 2$-Phenylethanol \\
-0.593 & $4:(Z)-3$-Hexenal & $11:(E)$-2-Hexen-1-ol \\
\hline
\end{tabular}

Table 2. Factor loadings $(>0.5)$, eigenvalues and cumulative proportion in factors 1 to 3 with mean GC area ratios in the two clones and correlation coefficients $(r)$ with sensory scores.

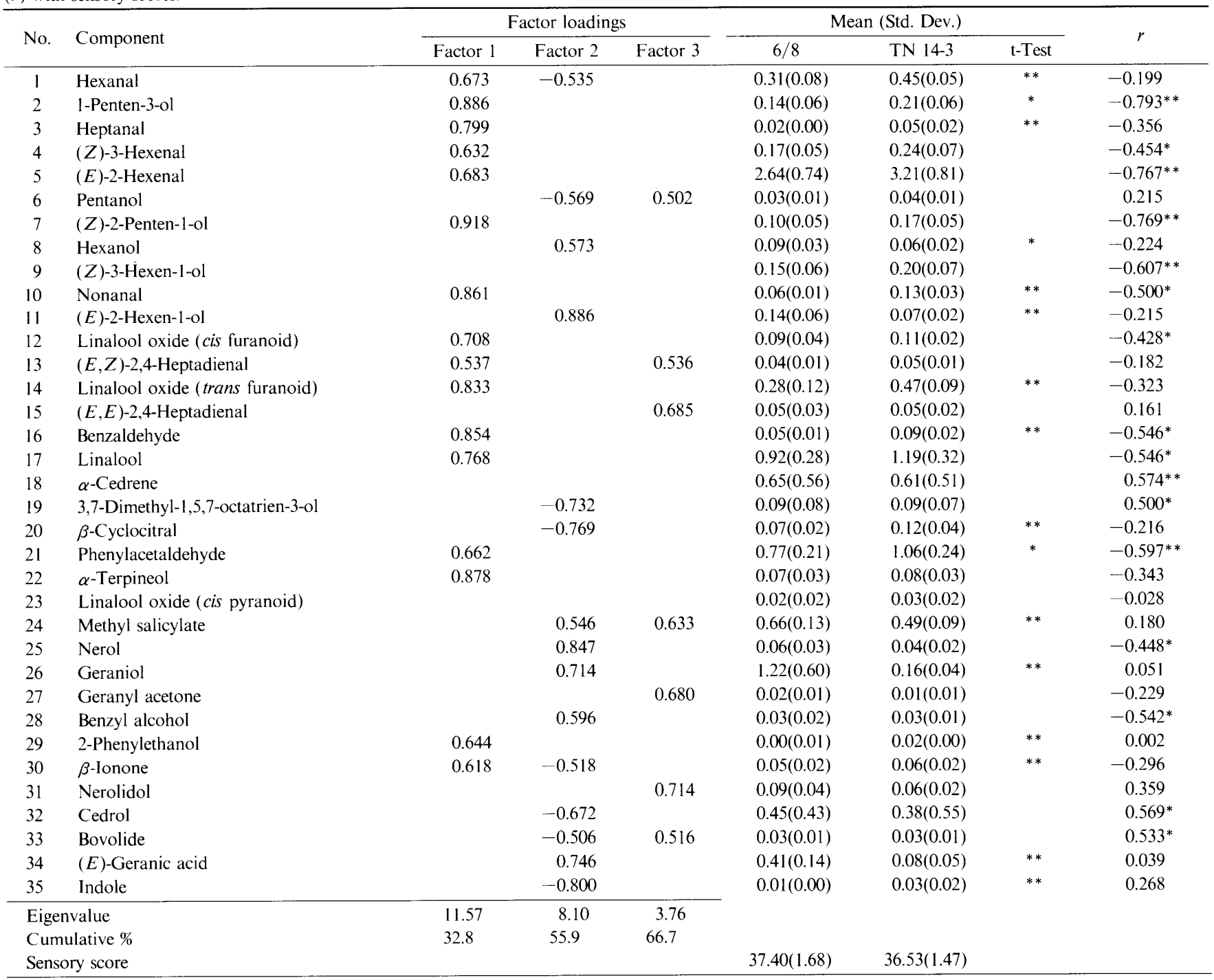

${ }^{*} P<0.05,{ }^{* *} P<0.01$. 
residuals of all deleted samples. This procedure was repeated until all samples were deleted at least once to calculate an MSEP (mean squared error of prediction) $=\Sigma\left(y_{i}-\hat{y}_{i}\right)^{2} / n$, where $y_{i}$ and $\hat{y}_{i}$ stand for $i$ th sensory score and the its predicted value, respectively. Next, PC 1 and PC 2 were used to calculate an MSEP. Thus, the number of PC was incremented by one until MSEP started increasing.

The stepwise MLR was performed by SPSS for Windows ver. 6.0. PLS and PCR were performed by UNSCRAMBLER ver. 5.3 (CAMO, Trondheim, Norway). All statistical analysis were performed using a DTK FEAT-5030 486 PC system with 8MB RAM (Datatech Enterprises, Taipei, Taiwan).

\section{Results and Discussion}

Mutual relationships of volatile components Many components were highly correlated to each other since most of the major components in black tea were oxidation products of nonvolatile components through the fermentation process utilizing enzymes originally contained in tea leaves. The highly correlated pairs of components are listed in Table 1. High correlations observed between aliphatic alcohols and/or aldehydes such as ( $Z$ )-2-penten-1-ol vs. 1-penten-3-ol and $(E)$-2-hexenal vs. 1-penten-3-ol can be easily ascribable to their conformity in starting materials, i.e., long chain unsaturated fatty acids such as linoleic, linolenic and palmitic acids (Wright \& Fishwick, 1979). However, terpene alcohols and their derivatives, i.e., geraniol and $(E)$-geranic acid, are originally produced by the hydrolysis of their glycosides (Yano et al., 1990). The oxidation of carotenoids is another important source of various ionones (Yamanishi, 1981). On the other hand, the highly negative correlation found between hexanal and $(E)$-2-hexen-1-ol indicates the existence of a trade-off relationship in their transformation pathway.

Factor analysis Eight factors accumulating $92.7 \%$ of the variance in the whole GC data were extracted from the $35 \times 16$ matrix by factor analysis. However, three initial factors, i.e., the first, second and third factors, collected $66.7 \%$

of the variance (Table 2). All heavily loaded components correlated positively to factor 1 . Among them, unsaturated aliphatic compounds eluted earlier, i.e., 1-penten-3-ol, $(E)$-2hexenal and ( $Z$ )-2-penten-1-ol, had a highly negative correlation to sensory scores. Consequently, samples assigned to smaller factor 1 scores were evaluated as better quality (Fig. 2). Significantly larger contents of hexanal, heptanal, nonanal and benzaldehyde in clone TN 14-3 than those in clone 6/8 made sample separation of the two clones possible. Concerning the factor 2 axis, on the other hand, much larger contents of geraniol and geranic acid in clone 6/8 (geraniol: 1.22, geranic acid: 0.41 ) than those in clone TN 14-3 (geraniol: 0.16, geranic acid: 0.08 ) explains the separation of the two groups each corresponding to clones $6 / 8$ and TN $14-3$, respectively (Table 2). This separation of samples does not necessarily reflect a difference in aroma qualities since most of the components loaded heavily on factor 2 did not highly correlate to the sensory scores. Although Yamanishi et al. (1989) reported that linalool and benzaldehyde greatly contribute to black tea quality, these correlation coefficients did not support their claims.

Thus the black tea samples belonging to the two clones were clearly separate. This separation suggested that volatile component patterns of the two clones were inherently different regardless of cultivated areas.

Clustering two clones Sample clusterings were different when the cluster analysis was applied to ratios of the GC peak areas which reflected absolute amounts of components and then to $Z$-scores in order to avoid being influenced by absolute component amounts. Of course, clusterings changed according to algorithms employed for measuring similarities or distances of samples and for merging the resulting subclusters.

As long as the Euclidean distance was being used, well ordered clustering did not form. However, well ordered clusterings were obtained when cosines of the vectors composed of $Z$-scores were calculated. Two large clusters, each composed of two clones, were found when Ward's method

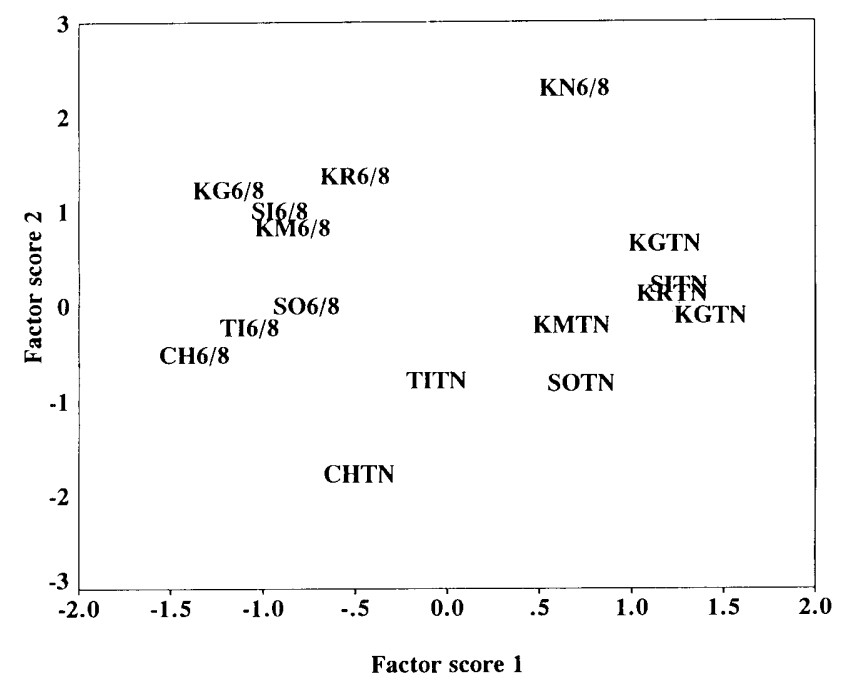

Fig. 2. Scatter plot of black tea samples on the basis of factors 1 and 2 .
(A)

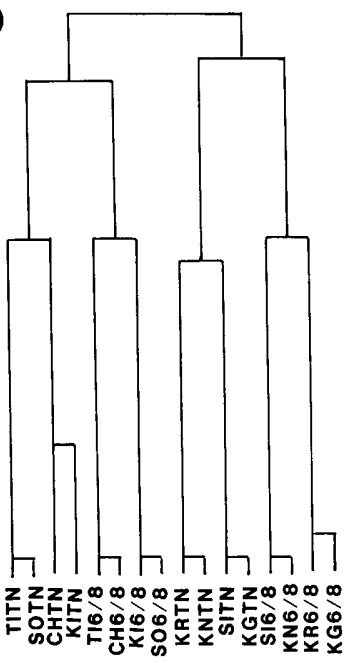

(B)

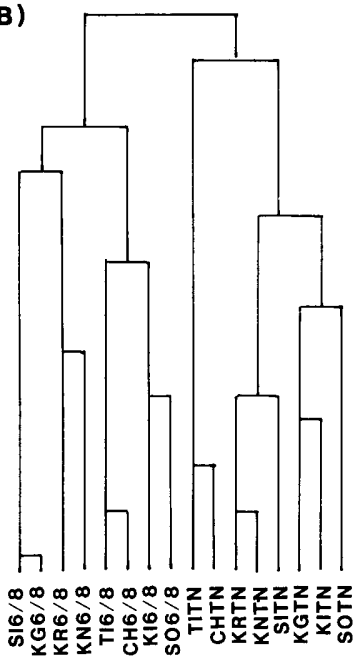

Fig. 3. Clustering of black tea samples based on the cosine similarity and Ward's method (A), and the cosine similarity and centroid method (B). 


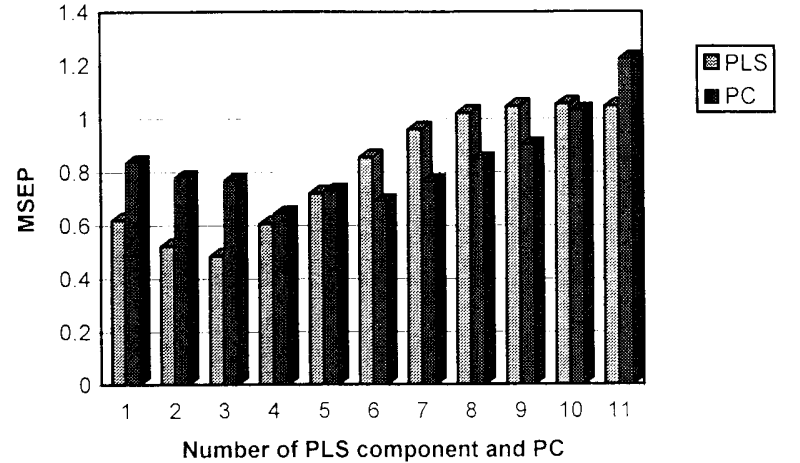

Fig. 4. MSEP calculated from cross-validation in PCR and PLS regression analyses.

was applied (Fig. 3A). However, each of the two large clusters split into two smaller clusters, both of them composed of two clones cultivated at four common areas. On the other hand, two large clusters, each corresponding to clones TN 14-3 and $6 / 8$, were formed when the centroid method was used (Fig. 3B).

Multivariate calibration The highest bivariate correlation with sensory scores shown by 1-penten-3-ol was -0.793 (Table 2$)$. This means that $62.9 \%\left(r^{2}=0.629\right)$ of the deviation in the sensory scores can be explained based on this unsaturated alcohol alone. However, higher predictive equations were needed for applying them to a practical assessment of black tea quality.

At first, stepwise MLR was used to calculate a predictive equation by selecting effective volatile components one by one. Peaks 2, 15 and 14 were selected as predictor variables for the multiple regression model (Eq. (4)) calculated at step 3.

$$
\begin{aligned}
y= & -26.379 \times \text { peak } 2(1 \text {-penten-3-ol }) \\
& +23.605 \times \text { peak } 15((E, E)-2,4 \text {-heptadienal }) \\
& +4.887 \times \text { peak } 14(\text { linalool oxide, trans furanoid }) \\
& +38.515
\end{aligned}
$$

The multiple correlation coefficients $(R)$ at steps 1, 2 and 3 were $0.793,0.862$ and 0.914 , respectively. $R^{2}$ expressing the explained ratio of deviation in sensory scores 4 was 0.835 at step 3 . However, $R$ and $R^{2}$ adjusted by the degrees of freedom were 0.891 and 0.794 (SPSS; Norusis, 1993) since the available number of samples was only 16 in this data set. Although peak 14 negatively correlated to sensory scores, a positive regression coefficient was calculated due to the multicollinearity existing between peaks 2 and 14. The necessary condition for changing the sign for the regression coefficient from the sign for the correlation with a sensory score is $r_{14, y}<r_{2,14} \times r_{2, y}$, regardless of their signs. Here, $r_{14, y}, r_{2,14}$ and $r_{2, y}$ indicate the correlation coefficients for peak 14 vs. sensory scores, peaks 2 vs. 14 and peak 2 vs. sensory scores, respectively. In this case, $r_{14, y}, r_{2,14}$ and $r_{2, y}$ were $-0.323,0.698$ and -0.793 , respectively. Thus, $r_{14, y}=0.323<r_{2,14} \times r_{2, y}=0.553$. As clearly shown by the contradiction between the two signs assigned for the multiple regression coefficient and bivariate regression coefficient due to the multicollinearity, application of MLR to strongly correlating variables causes serious problems.

MLR is sometimes called a hard modelling method because only a useful sub-set of variables is used to calculate equations. On the other hand, PCR and PLS are called soft

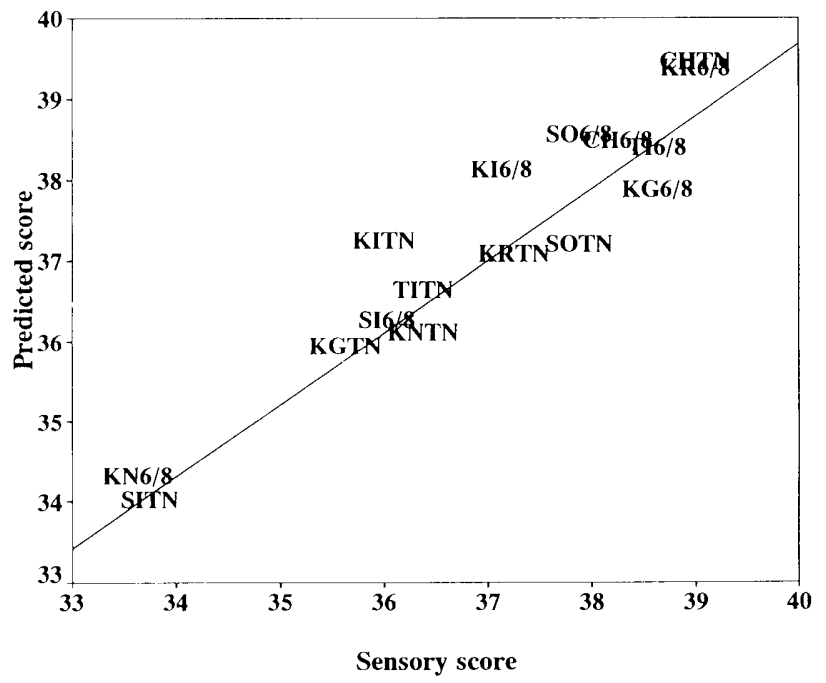

Fig. 5. Relationship between sensory scores and their predicted values by PLS regression model.

modelling methods in which whole predictor variables are used for prediction. Both PCR and PLS can avoid multicollinearity problems since orthogonal or non-correlating principal components (PC) or PLS components are used as predictors. The MSEP for the model composed of PC 1 was 0.837 and then decreased to $0.780,0.770$ and 0.646 when PCs 2-4 were added one by one into models but increased to 0.731 when PC 5 was added (Fig. 4). Thus, the regression model composed of four PCs suggested to be the best from crossvalidation. However, $R$ was only 0.811 in the regression model composed of the four PCs.

The MSEP for the PLS model composed of the 1st PLS component was 0.621 and decreased to 0.523 and 0.485 by adding the 2 nd and 3 rd PLS components but increased to 0.606 when the 4 th PLS component was added (Fig. 4). $R$ and $R^{2}$ of the best PLS model decided by cross-validation were 0.946 and 0.895 , respectively (Fig. 5). Although $R$ and $R^{2}$ decreased to 0.932 and 0.869 when adjusted by degrees of freedom, these values were the largest among those calculated using the three multivariate calibration methods. Factor loadings of the 1st and 2nd PLS components for 35 volatile components and sensory scores are shown in Fig. 6. Peaks 18, 19, 32 and 33 locate closely to the "sensory" scores in the scatter plot. The distance of the components from the "sensory" scores in the scatter plot indicates that $\alpha$-cedrene, 3,7-dimethyl-1,5,7-octatrien-3-ol, cedrol and bovolide highly correlate to the sensory quality of black tea. On the other hand, the locations of peaks 2, 7, 9, 17 and 21 show their opposite effects on the sensory quality. The abundance of these components may indicate poor quality in black tea aroma. Factor loadings in PLS and correlation coefficients of bivariate regression models coincided in their correlations to sensory scores.

\section{Conclusions}

Factor analysis and cluster analysis succeeded in classifying black tea samples according to clones and geographic origins based on their volatile components. Benzaldehyde, linalool and their oxides have been claimed as the components 


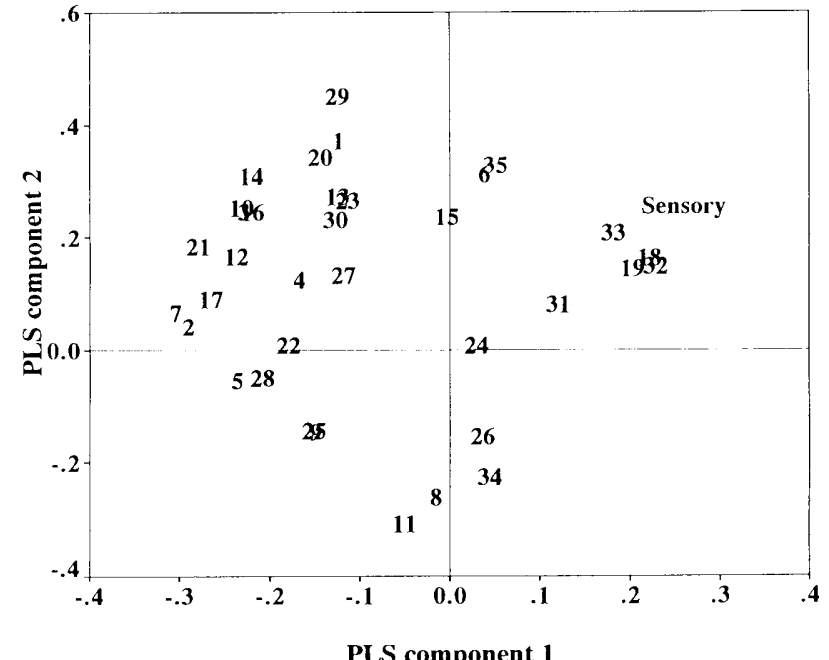

Fig. 6. Factor loading plot of volatile components with sensory score in PLS components 1 and 2 .

contributing to tea quality but correlation coefficients suggested the opposite tendency. Multivariate calibration methods were useful to calculate equations to predict sensory scores of black tea samples from their volatile components. Multicollinearity failed to obtain reliable equations from MLR but this problem can be avoided in both PCR and PLS analyses. Most of the aliphatic aldehydes and alcohols as well as linalool and linalool oxides showed strongly negative effects on tea quality. All results obtained from a chemometrics analysis showed clearer aspects than those suggested in the previous papers where somewhat arbitrary selections and assessments of components were made. However, chemometrics methodologies should be applied to a larger number of samples in order to obtain more generalized information.

\section{References}

Aishima, T. and Nakai, S. (1991). Chemometrics in flavor research. Food Rev. Int., 7, 33-101.

Flament, I. (1989). Coffee, cocoa, and tea. Food Rev. Int., 5, 317-414.

Grant, A., Flanklin, J.G. and Daviea, A.M.C. (1988). Near infrared analysis: the use of multivariate statistics for investigation of variables in sample preparation and presentation of tea leaf. $J$. Sci. Food Agric., 42, 129-139.

Guth, H. and Grosch, W. (1993). Identification of potent odourants in static headspace samples of green and black tea powders on the basis of aroma extract dilution analysis (AEDA). Flavour Fragrance J., 8, 173-178.
Mahanata, P.K., Baruah, S., Owuor, P.O. and Murai, T. (1988). Flavour volatile of Assam black teas manufactured from different plucking standards and orthodox teas manufactured from different altitudes of Darjeeling. J. Sci. Food Agric., 45, 317-324.

Nakagawa, M., Anan, T. and Ishima, N. (1981). Relations of green tea taste to chemical composition. Bull. Natl. Res. Inst. Tea., 17, 69123 (in Japanese).

Norusis, M.J. (1993). "SPSS for Windows. Professional Statistics Release 6.0." SPSS, Chicago, pp. 83-109.

Osborne, B.G. and Fearn, T. (1988). Discriminant analysis of black tea by near infrared reflectance spectroscopy. Food Chem., 29, 233 238.

Owuor, P.O. (1992). Comparison of gas chromatographic volatile profiling methods for assessing the flavour quality of Kenyan black teas. J. Sci. Food Agric., 59, 189-197.

Owuor, P.O., Othieno, C.O. and Takeo, T. (1989). Effects of maceration method on the chemical composition and quality of clonal black teas. J. Sci. Food Agric., 49, 87-94.

Owuor, P.O., Tsushida, T., Horita, H. and Murai, T. (1988). Effects of geographical area of production on the composition of the volatile fiavour compounds in Kenyan clonal black CTC teas. Exp. Agric., 24, 227-235.

Sharaf, M.A., Illman, D.L. and Kowalski, B.R. (1986). "Chemometrics." John Wiley \& Sons, New York, pp. 254-255.

Togari, N., Kobayashi, A. and Aishima, T. (1995a). Relating sensory properties of tea aroma to gas chromatographic data by chemometric calibration methods. Food Res. Int., In press.

Togari, N., Kobayashi, A. and Aishima, T. (1995b). Pattern recognition applied to gas chromatographic profiles of volatile components in three tea categories. Food Res. Int., In press.

Tomlins, K.I. and Gay, C. (1994). Prediction of quality and origin of black tea and pine resin samples from chromatographic and sensory information: evaluation of neural networks. Food Chem., 50, 157165.

Wickremasinghe, R.L., Wick, E.L. and Yamanishi, T. (1973). Gas chromatographic mass spectrometric analysis of flavoury and non-flavoury Ceylon black tea aroma concentrates prepared by different methods. J. Chromatogr., 79, 75-80.

Wright, A. and Fishwick, M.J. (1979). Lipid degradation during manufacture of black tea. Phytochemistry, 18, 1511-1513.

Yamanishi, T. (1981). Tea, coffee, cocoa and other beverages. In "Flavor Research-Recent Advances," ed. by R. Teranishi, R.A. Frath and H. Sugisawa. Marcel Dekker, New York, pp. 231-304.

Yamanishi, T., Wickeremasinghe, R.L. and Perera, K.P.C.W. (1978). Studies on the flavour and quality of tea. 3-Gas chromatographic analysis of aroma complex. Tea $Q$., 39, 81-86.

Yamanishi, T., Bothejyu, W.S. and De Silva, J.M. (1989). An index for assessing the quality of Uva seasonal black tea. Sri Lanka J. Tea Sci., 58, 40-49.

Yano, M., Okada, K., Kubota, K. and Kobayashi, A. (1990). Studies on the precursors of monoterpene alcohols in tea leaves. Agric. Biol. Chem., 54, 1023-1028. 